\title{
Kulturkritik, phénoménologie et subjectivité à l'âge de la technique
}

Par MANLIO IOFRIDA

Università di Bologna

Je trouve très intéressant le fait que les travaux de ce séminaire soient organisés autour de la contraposition entre deux pôles : d'un côté, celui du Kulturpessimismus, ou de la Kulturkritik, de la critique envers la modernité et la société industrielle, de l'autre, celui des réponses face à la société bourgeoise, qui pourraient bien être qualifiées de "philomodernes» ou « modernisantes » - je pense par là aux conceptions selon lesquelles la technique, la massification ainsi que le développement des moyens de communication sont de véritables ressources, des données de fait qu'il faut accepter, voire mettre à profit du point de vue aussi bien politique qu'esthétique. La première ligne est épousée par Schopenhauer, par un certain Nietzsche (Nietzsche a toujours plusieurs facettes, comme vous le savez), par Jakob Burckhardt, et il s'agit donc d'une ligne essentiellement (mais pas exclusivement) allemande, apparentée à l'idée outre-rhénane de Kultur; la seconde ligne est celle, française, de la civilisation, même si, en ce sens également, elle a bien ses représentants allemands : il s'agit de la position issue de l'expérience politique de la grande Révolution, mais aussi de la révolution industrielle. C'est tout le XIX ${ }^{\mathrm{e}}$ siècle français, avec son mythe de la technologie et de ses infinies révolutions politiques, c'est le siècle durant lequel des romantiques si différents les uns des autres que Hugo, Baudelaire et Rimbaud peuvent se retrouver dans l'éloge de la ville moderne et de la modernité. Sur ces deux grands axes, où pouvons-nous donc placer la phénoménologie de Husserl, et l'interprétation qu'en a donnée Maurice Merleau-Ponty en France, interprétation sur laquelle portera en grande partie la suite de mon propos ? Cette question n'appelle pas une réponse facile, directe et unilatérale. Concernant Husserl par exemple, sa position face à l'alternative Kulturpessimismus-modernisation n'est pas du tout univoque : à 
n'en pas douter, sa pensée, avant tout celle contenue dans la Crise des sciences européennes, s'enracine dans une philosophie de la vie qui appartient à la première ligne, mais au même titre, il n'y a aucun doute sur le fait que la critique de la science placée au centre du dernier grand ouvrage husserlien ne représente pas un refus de la modernité et de la rationalité technique. En outre, la position husserlienne est dépourvue de cet élitisme que l'on retrouve comme l'un des traits caractéristiques du Kulturpessimismus. Car en effet, on ne peut oublier que ces deux lignes dont nous parlons représentent aussi des modèles politiques en grande partie opposés : le pessimisme culturel nourrit une aversion féroce envers la démocratie et la société de masse, tandis que l'autre position, fréquemment liée au marxisme ainsi qu'à la France révolutionnaire, est très favorable à la démocratie et ne perçoit dans la massification aucun risque de décadence. De ce point de vue, la position de Merleau-Ponty est encore plus complexe que celle de Husserl : le penseur français est en effet profondément enraciné, sous divers aspects, dans la culture française, mais il a aussi été pendant quelques années un des tenants importants du marxisme français; de plus, Merleau-Ponty a fait confluer l'idée bergsonienne de vie avec les positions husserliennes de la Crise, en laissant émerger cette recherche sur l'idée de nature sur laquelle je reviendrai plus avant; entre Kultur et Civilisation, entre France et Allemagne, entre démocratie et élitisme, où placerons-nous la réflexion merleaupontienne sur la nature, sur l'histoire et sur le marxisme (dont il refusera certains aspects non secondaires, mais en maintiendra d'autres dont l'importance n'est pas moindre) ? Si je soulève ces interrogations, et si je me réfère à ces auteurs, c'est bien parce que ces questionnements - et je renvoie ici aux questions soulevées par ce séminaire - appartiennent à notre présent : nous avons d'une part assisté, au cours des deux dernières décennies, à une nouvelle explosion des contradictions de la société industrielle de masse, ou capitaliste, avec le lot de problèmes, désormais classiques, qui vont de pair: homogénéisation, imposition de clichés, culture massifiée frôlant souvent la barbarie - et il me semble que ce que nous, italiens, vivons avec Berlusconi en est un exemple très clair au niveau international ; d'autre part, pouvons-nous accepter la réponse élitiste du Kulturpessimismus à ces problèmes, qui engendre à partir de la critique à l'égard de la société de masse une remise en question de la notion de démocratie et de la valeur de l'égalité comme héritage de la Révolution française ? Démocratie, science et technologie sont-elles responsables de la crise de civilisation que nous traversons ? Ou au contraire, est-il possible de construire un appareil critique de la modernité moins absolu, aux accents moins pessimistes, et plus ouvert à une vision moins négative de la technologie ? Sur ce point il faut néanmoins 
introduire, par rapport aux deux lignes dont nous avons parlé jusqu'à maintenant, une troisième position: celle-ci caractérise notre présent de manière plus spécifique car elle a acquis une place prédominante durant les vingt dernières années : $j$ 'entends ici la question de l'écologie. Cette dernière, eu égard à la Kulturkritik et, plus encore face au modernisme, est un élément complètement nouveau: il va de soi que l'idée de civilisation est l'exact contraire d'une conception écologique mais, d'un autre côté, la conception de la Kultur, en substance, met principalement l'accent sur le moment de l'activité, sur la figure de l'artiste génial qui imprime sa forme sur la nature et, tout au moins sur un premier niveau, n'a ainsi pas grand chose à voir avec l'idée environnementaliste. Le constat d'une civilisation industrielle ayant atteint ses limites, tout comme l'idée désormais écartée d'un progrès infini de la production, voilà des données de fait qui, au moins à une échelle de masse, ne sont devenues claires que récemment. C'est à partir de ce nouvel horizon que la philosophie de Merleau-Ponty se montre encore à mon sens d'une actualité remarquable. En effet, la lecture de Husserl livrée par le philosophe français à la fin des années 1950, a abouti à une réflexion sur l'idée de nature - les cours dispensés par Merleau-Ponty entre 1956 et 1960 en font preuve - qui introduit des concepts nouveaux par rapport aux deux lignes que nous citions précédemment. Il faut alors à mon avis bien réfléchir sur la position merleau-pontienne, excentrée de points de vue divers, comme je le disais plus haut: Merleau-Ponty est assurément un fils de la civilisation et il appartient à la tradition de la grande Révolution, mais en même temps, il s'enracine dans plusieurs couches de la Kultur allemande. Il suffit de rappeler que sa pensée est tributaire des idées d'intellectuels comme Goldstein, Koffka et Wertheimer et qu'elle contient l'empreinte des philosophies de Husserl, Max Scheler et Martin Heidegger. Sur le versant français de Merleau-Ponty, il faudrait encore évoquer le rapport profond qui le lie à des personnages comme Proust et Cézanne, lesquels sont en un certain sens l'expression la plus mûre de ce $\mathrm{XIX}^{\mathrm{e}}$ siècle français qui fut bien celui d'une certaine modernité. N'oublions pas également son attention au cinéma - art lié, plus que les autres, à la technologie, à la société de masse et à la modernité et dont l'invention ne fut certes pas fortuite en France à la fin de ce même XIX ${ }^{\mathrm{e}}$ siècle. Comment alors, face à ces questions, le paradigme merleau-pontien de la corporéité — pivot de tout son discours - se situe-til ? Il s'agit pour nous d'une question fondamentale, car c'est à partir de cette idée du corps que Merleau-Ponty s'achemine vers une conception propre de la vie et de la nature ; corps, vie et nature sont ainsi les concepts qui, à partir de la nouvelle centralité de la question écologique, se révèleront fondamentaux pour la tentative de penser philosophiquement notre situation actuelle. 
Je tenterai donc par la suite d'approfondir un peu ce noyau conceptuel, en insistant surtout sur la question de l'activité et de la passivité dans la reprise de Husserl par Merleau-Ponty. De plus, je m'efforcerai de documenter très brièvement la manière dont ces idées merleau-pontiennes de corps et de nature ont été reprises ces dernières années dans plusieurs champs appartenant aux sciences humaines.

Dans son introduction, Rudy Steinmetz évoque les positions de Derrida, de Foucault et aussi de la problématisation de la modernité mise en œuvre par ces auteurs ${ }^{1}$. Il me semble essentiel, avant de nous pencher sur Merleau-Ponty, de m'arrêter un peu sur le poststructuralisme et sur le postmodernisme, qui ont été les deux mouvements culturels et philosophiques dominants, se succédant et s'entrecroisant, sur la scène philosophique de la seconde moitié du $\mathrm{XX}^{\mathrm{e}}$ siècle. Concernant le poststructuralisme, j'entends par ce terme, d'ailleurs très vague, la pensée française des années 1960, c'est-à-dire, essentiellement, les philosophies respectives de Foucault, Deleuze et Derrida ; quant au postmodernisme, je me réfère à la vague philosophique qui s'est affirmée dans les années 1980 ; il faut à mon avis jeter un regard sur ces mouvements car nous vivons aujourd'hui dans le vide qu'ils ont laissé derrière eux : nous sommes placés, pour ainsi dire, dans le mouvement de ressac du poststructuralisme et du postmodernisme ; il convient alors de voir, sous les angles qui nous intéressent, quel est l'héritage qu'ils nous ont laissé.

Donc, d'abord, le poststructuralisme. Dans un sens très large, il comprend aussi le structuralisme. Or, la question que je voudrais poser est la suivante : qu'a été la culture des années 1960 et quelle signification recouvre-t-elle aujourd'hui pour nous? La première réponse que je donnerais à cette question est que la culture de cet âge a été la dernière culture hégémonique exprimée par l'Europe, cette Europe qui, après la Seconde Guerre Mondiale, était devenue marginale par rapport aux deux blocs alors constitués par les États-Unis d'une part et l'URSS de l'autre. Il n'est pas difficile de voir aujourd'hui que la culture française des années 1960 a été une tentative de proposer une alternative aux deux grands modèles politiques, économiques et culturels des deux grandes puissances, deux modèles qui étaient au même titre centrés sur la valeur de la production et du travail collectif. Mais, en se démarquant du communisme et du capitalisme, quelles valeurs alternatives,

${ }^{1} C f$. Rudy Steinmetz, « Présentation » de ce Séminaire. 
quel modèle allait proposer cette culture des années 1960 ? Les valeurs de la Kultur, celles de la Civilisation? Ou plutôt un certain primitivisme, qui émergeait d'une culture anthropologique comme celle de Claude LéviStrauss ? La réponse à une telle interrogation n'est pas simple, pour plusieurs raisons : d'abord parce que la position de cette culture face au phénomène du néocapitalisme, c'est-à-dire face à la dernière grande révolution industrielle que nous avons connue, et en plein déroulement à l'époque, a été une réponse ambiguë: elle n'acceptait pas les valeurs du capitalisme, mais le vaste processus de modernisation qui touchait alors le monde occidental bénéficiait d'un jugement positif. Je veux dire qu'en fait, dans l'ensemble, une position de pessimisme culturel, une critique radicale de la civilisation moderne ne passe pas à Paris ; au contraire, se forme un horizon de grandes espérances, de confiance dans le futur : le côté obscur du processus de modernisation semble cacher une face lumineuse et positive. Cette attitude très complexe, ou même contradictoire, se retrouve aussi sur le problème crucial de la révolution: la culture française des années 1960 a l'un de ses présupposés (même pour un révolutionnaire marxiste comme l'était Louis Althusser) dans la critique de la révolution soviétique, dans la crise qui avait frappé en 1956 le bloc comme le marxisme soviétique. Tout le projet du structuralisme et du poststructuralisme doit être évalué sous cet angle de l'échec de l'idée de révolution: l'histoire ne peut être rapportée à des choix transparents et volontaires, il y a des noyaux structurels qui résistent, ne se laissant nullement anéantir par la volonté révolutionnaire. Donc, d'un certain point de vue, une critique conséquente de la grande idée de la révolution était implicite dans les positions de Foucault, de Derrida, même de Gilles Deleuze. Et pourtant, au moins dans les années 1960, ces auteurs adoptent sur ce point une position contradictoire: la critique envers la révolution soviétique s'accompagne de l'idée qu'un changement total est en train de se produire, que le monde est en train de se transformer d'un bout à l'autre, que tout le vieux monde de la civilisation humaniste touche à sa fin... Vous avez déjà compris qu'en disant ceci, c'est à ce manifeste des années 1960 représenté par Les Mots et les choses de Michel Foucault ${ }^{1}$ que je me réfère, et donc par là à sa proclamation de la mort de l'homme. Il y a avait dans certains passages de ce grand livre une sorte d'espérance - ou de crainte, ce qui revient au même - apocalyptique, l'idée d'être au début d'une ère complètement nouvelle, mais même si vous prenez un texte bien différent comme De la grammatologie de Jacques Derrida ${ }^{2}$, vous retrouverez ces

${ }^{1}$ Paris, Éditions Gallimard, 1966.
${ }^{2}$ Paris, Éditions de Minuit, 1967. 
mêmes accents. Mais d'où provenait donc l'idée qu'un nouveau monde était en train de naître? C'était naturellement une manière de réagir et d'interpréter le néocapitalisme. Je posais plus haut que la modernisation semblait, pour cette génération intellectuelle, cacher une face lumineuse et positive. Pour le dire de la manière la plus claire possible, prenons le final des Mots et les choses: le jugement de Foucault sur la technicisation, l'affirmation des sciences humaines, la mort de l'homme est-il positif ou négatif? Difficile de répondre avec un oui ou un non. Il est évident que Foucault n'accepte pas la réduction du savoir aux sciences humaines, c'est-àdire à la machine de l'industrie culturelle en voie d'édification par le néocapitalisme; mais il est aussi certain que sa posture n'est pas antimoderne, romantique : elle renferme l'idée que derrière le grand processus de modernisation et son masque nihiliste, sa destruction de toutes les valeurs, se cache quelque chose de complètement autre: cette nouvelle littérature, ce Langage Absolu qui n'ont rien à voir avec la société bourgeoise et son utilitarisme borné et qui peuvent être le fondement d'une nouvelle société. La perspective marxiste d'une révolution comme instrument de libération n'avait donné aucun signe de réussite; face à cet échec, il fallait plutôt déceler dans le processus de modernisation néocapitaliste un levier qui aurait produit un monde intrinsèquement anticapitaliste. Voilà la position de Foucault sur le néocapitalisme et la modernisation, position qui est caractéristique de toute sa génération, laquelle est une génération ayant refusé la révolution soviétique. Mais, sur la base de ce refus, elle n'a tiré comme conséquence ni la nécessité d'une révision du paradigme moderne, ni une critique du productivisme et de la technologie commune au capitalisme et au socialisme soviétique, ni l'espérance en un quelconque renversement total, en une révolution (différente de la révolution soviétique). Nous voici donc parvenus aux limites de cette culture, à sa solidarité avec le projet moderne de maîtrise et d'exploitation de la nature.

Avant d'amorcer ce discours, qui conduira à l'examen de la position merleau-pontienne très divergente à ce sujet et pour conclure cette reconsidération nécessairement très rapide et simplifiée de la culture française des années 1960, je voudrais m'arrêter un moment sur un aspect totalement différent, c'est-à-dire sur ce que, de manière encore une fois trop simplifiée, l'on peut qualifier de "primitivisme ", lequel avait son point de repère essentiel dans l'œuvre de Claude Lévi-Strauss. Par rapport aux positions de Foucault et de Derrida à peine mentionnées, la pensée de Lévi-Strauss se trouve aux antipodes - et vous vous rappellerez, à ce propos, la polémique 
cuisante qui s'est produite entre Derrida et Lévi-Strauss ${ }^{1}$ : chez ce dernier, le refus de la civilisation, au nom d'un certain rousseauisme, était très net et la critique sévère à l'égard de l'Occident se rattachait à un rejet du processus $\mathrm{d}^{\prime}$ industrialisation capitaliste ${ }^{2}$. Au-delà du caractère philosophiquement simpliste de ces positions, il faut bien en mesurer le poids d'un point de vue politique : en effet, la crise du colonialisme se traduisait à travers une vague puissante qui insistait avec force sur la dimension d'actualité, voire même de supériorité, des cultures primitives. Le mythe de la Chine, une civilisation paysanne qui avait rompu avec le modèle stalinien et soviétique du socialisme, le mythe de Cuba... étaient, nous le savons désormais, des mythes, mais leur circulation dans la vielle Europe, secondée par la diffusion de Tristes Tropiques de Lévi-Strauss ${ }^{3}$, impliquait une profonde autocritique de la civilisation européenne, ainsi que l'idée d'un nouveau pacte entre nature et culture qui préludait déjà à l'écologie.

La leçon de cette grande culture des années 1960 est donc bien loin d'être cohérente et univoque : entre Foucault, Derrida, Althusser, Deleuze d'un côté, et Lévi-Strauss de l'autre, on voit se reproduire une sorte de polarité opposant culturalisme absolu et naturalisme ingénu. Mais même sur les autres questions, on peine à retracer une position claire : la révolution était-elle critiquée ou y avait-il plutôt attente d'un changement encore plus absolu? Et sur la technique et les moyens de communication, quelle était alors la position, face à celle de l'École de Francfort, qui à cette époque avait déjà sur ces thèmes un programme très clair? L'identification de ces difficultés — définies comme telles bien sûr du point de vue que nous adoptons, s'agissant de problèmes auxquels nous sommes confrontés dans le présent - ne comporte pas en ce qui me concerne, un changement dans le jugement de valeur sur cette culture : celle-ci a été, je le répète, la dernière grande époque de la philosophie se trouvant derrière nous ; il faudrait peutêtre juste préciser les termes, autrement dit, elle a été la dernière grande

${ }^{1}$ Cf. Jacques Derrida, La Violence de la lettre : de Lévi-Strauss à Rousseau, in Id., De la grammatologie, op. cit., Deuxième partie, chap. 1 (une première version de ce texte a été publiée dans Les Cahiers de l'Analyse, vol. 4, 1966, sous le titre « Nature, culture, écriture: la violence de la lettre de Lévi-Strauss à Rousseau »). $C f$. également Claude Lévi-Strauss, "Une lettre à propos de "Lévi-Strauss dans le dixhuitième siècle" », dans Les Cahiers de l'Analyse, vol. 8, 1967, p. 89-90.

${ }^{2}$ Je suis bien conscient qu'il n'est pas possible de placer sous cette étiquette la totalité de l'œuvre de Lévi-Strauss : mon propos renvoie davantage à un certain "lévi-straussisme » qui fut l'un des courants dominants de la culture des années 1960.

${ }^{3}$ Paris, Éditions Plon, 1955. 
époque de la philosophie moderne, d'une philosophie qui se sentait et se voulait moderne. Il est alors naturel de passer maintenant au discours sur le postmodernisme.

À la fin des années 1980, comme on le sait bien, le climat politique et culturel a brusquement changé et l'avènement du postmodernisme, qui a dominé les vingt années successives, en a été le signe. Je précise que je parlerai aussi à propos du postmodernisme d'une sorte de «type idéal », tout comme je le faisais concernant le "poststructuralisme» dont j'ai choisi certains éléments capitaux pour mon propos; en conséquence, je me concentrerai sur la dimension philosophique et politique de ce mouvement, en me référant essentiellement au célèbre texte sur la condition postmoderne de J.-F. Lyotard ${ }^{1}$, qui fut le manifeste « philosophique » d'un mouvement par ailleurs plurivoque et complexe, c'est-à-dire riche de facettes sociologiques, économiques et esthétiques très variées. Il va également de soi qu'il existe en fait entre le postmodernisme et les positions des poststructuralistes une infinité d'échanges et de points communs - à ce propos, il suffit de considérer le profil de J.-F. Lyotard lui-même, qui est un représentant mineur de cette grande génération des années 1960, et de rappeler que même Foucault, Deleuze, Derrida ont été et sont encore aujourd'hui fréquemment associés au postmodernisme. Néanmoins, cela n'empêche pas de voir dans ce dernier le signe d'une nouvelle période : signe de la crise du bloc soviétique et de son écroulement, de l'avènement du néolibéralisme et du crépuscule du keynésianisme, et enfin de la prédominance écrasante des États-Unis qui a marqué tout cet âge. En effet, le postmoderne en philosophie est la première conception qui marque l'inversion de la relation entre Europe et États-Unis : si le poststructuralisme a été la dernière grande saison de l'hégémonie philosophique européenne, le postmodernisme est un signe de l'hégémonie des États-Unis. Essayez de lire La Condition postmoderne de ce point de vue : il vous paraîtra évident que le livre sonne le glas de la vieille culture européenne, de la civilisation française de la Révolution et de la Kultur allemande de Goethe et de Humboldt - et, implicitement, du poststructuralisme qui en avait été la dernière grande expression. Au centre du livre de Lyotard - je n'en donnerai donc qu'une esquisse très rapide - nous trouvons la grande révolution technologique qui au cours de ces années 1980

${ }^{1}$ Cf. Jean-François Lyotard, La Condition postmoderne. Rapport sur le savoir, Paris, Éditions de Minuit, 1979. 
s'irradiait dans tout le système capitaliste, une révolution portée par l'application de l'informatique à la production. À l'instar de nombreux sociologues, Lyotard interprétait ce phénomène en avançant l'idée que la production de marchandises matérielles, dotées d'une valeur d'usage, serait désormais secondaire, tandis que les marchandises immatérielles n'ayant pour contenu que l'information auraient pris le dessus. En renvoyant, implicitement, à l'idée de fin de l'histoire d'Alexandre Kojève, Lyotard en concluait que le capitalisme contemporain aurait complètement dompté la nature : la $\tau \dot{z} \chi v \eta$ (technè) — c'était aussi une leçon heideggérienne qui venait d'être confirmée - aurait réduit la réalité au langage de l'informatique, à une succession de 0 et de 1 , et c'était sur ce terrain du langage - des choix hétérodoxes à ce niveau, des paralogies et jeux linguistiques de Wittgenstein et de Feyerabend - que se déplaçaient toutes les contradictions et les possibilités d'opposition à la logique du profit capitaliste. Nous avons vu que la question de la nature, des limites de la technicisation du monde n'était pas au centre du paradigme poststructuraliste; or, il est évident qu'il l'était encore moins dans la vision postmoderne qui était, au contraire, complètement dominée par une conception prométhéique de la relation homme-nature. Il n'est donc pas étonnant que le postmoderne ait aussi été l'époque de l'obsession technologique, du Cyberpunk, époque marquée par des films comme Matrix ou Existenz, où l'on assiste à une véritable invasion du corps et de la nature par la technologie; mais c'est aussi le temps de l'illusion consistant à croire dans la capacité de cette invasion de conduire à une joyeuse libération, un corps nouveau libéré de ses chaînes et de ses limites, selon la plus classique vision faustienne... Cette illusion s'accompagnait de la pensée que les conflits sociaux n'étaient plus à l'ordre du jour : l'écroulement du marxisme et de l'idée de classe obscurcissait la voie du nouveau capitalisme, néolibéral, lequel accomplissait une accentuation des inégalités à la fois entre Nations et à l'intérieur des Nations mêmes, un capitalisme appauvrissant tant la classe laborieuse que la classe moyenne... Il n'y a pas de doute - la crise actuelle est très éclairante à ce propos - sur le fait que le postmoderne, considéré de ce point de vue, ait été une idéologie, dans le sens marxien le plus classique, une idéologie du capitalisme néolibéral et financier. Je ne veux pas dire qu'il s'est agi uniquement de cela — et d'ailleurs, le concept marxien d'idéologie n'est pas si innocent, il n'est pas non plus de nos jours maniable sans équivoques. Le postmodernisme n'a pas été une conception unilatéralement conservatrice ou idéologique; il y avait en effet en son sein de nombreuses instances positives et aujourd'hui encore vivantes. En premier lieu, il a mis au centre ce moment de l'individualité avec son irréductibilité, qui comportait une prise en charge nouvelle 
de la question de la subjectivité : cette question était restée problématique dans le poststructuralisme (exception faite du Foucault de la maturité, qui fut en effet le seul à s'être mesuré aux questions posées par le postmodernisme). La mort de l'homme avec laquelle Foucault concluait Les Mots et les choses, la discussion engagée par Derrida sur l'humanisme dans «Les Fins de l'homme $»^{1}$, la destruction du sujet contenue dans un texte comme l'AntiEdipe de Deleuze et Guattari ${ }^{2}$ ne donnaient pas d'instruments suffisants pour penser le grand retour de l'individu représenté par l'ère néolibérale. De ce point de vue, le poststructuralisme avait tenté d'opposer au capitalisme un moment collectif, celui des structures impersonnelles, et il s'agissait d'un collectif différent de celui des structures hégéliennes apparentées au modèle socialiste, soviétique ou non, mais cette élaboration était de toute évidence insuffisante. Ici le texte de Lyotard posait courageusement un problème - je le répète, celui de repenser le statut de l'individu et du sujet et par conséquent de la relation individu-société, ou, en termes plus philosophiques, de la relation soi-même-autrui; toute la réflexion de Foucault analysant le paradigme de la biopolitique, mais aussi l'herméneutique du soi et la réintroduction d'un certain sujet sont sans nul doute traversées par une discussion implicite avec La Condition postmoderne de Lyotard et le problème du statut du sujet et de la relation avec l'autre est, il va sans dire, au centre de notre réflexion aujourd'hui. Dans le texte de Lyotard un autre aspect bien vivant, et lié au précédent réside dans la centralité de la question de la différence, de la diversité, de l'hétérogénéité irréductible. De ce point de vue, le postmodernisme a bien été un mouvement très progressif, car il a porté au premier plan ce thème de la différence qui était fondamental pour bien des mouvements au niveau politique et social : je pense par exemple tout d'abord au féminisme, mais aussi à toutes les cultures postcoloniales ou, tout simplement, non occidentales, voire se trouvant aux confins de l'Europe et de l'Occident. De nos jours, cette variété de traditions est en pleine explosion, mais il faut garder à l'esprit que, déjà à l'époque postmoderne, sous le chapeau de la puissance unique américaine, bien des tensions et bien des forces étaient latentes et allaient dans des directions contraires à celles qui prédominaient.

${ }^{1}$ Cf. Jacques Derrida, Marges. De la philosophie, Paris, Éditions de Minuit, 1972, p. 129 sq. (il s'agit du texte d'une conférence tenue en 1968).

${ }^{2}$ Paris, Éditions de Minuit, 1972. 
Mais aujourd'hui, où sommes-nous donc ? Je ne crois pas que l'assertion selon laquelle l'époque postmoderne est désormais achevée ait besoin de trop de justifications: nous tous, je crois, en sommes très conscients. Le 11 septembre 2001 et ses suites en ont été la marque sanglante. Mais quelles sont les caractéristiques de cette nouvelle époque ? En premier lieu, cette date a bien donné le signal que nous ne trouvions pas dans cette époque de la rationalité accomplie annoncée par Francis Fukuyama en reprenant la leçon de Kojève ; l'histoire ne s'achève, ni par le bien ni par le mal ; elle n'est, surtout, pas achevée au sens positif d'une survivance de traditions et des cultures qui résistent à la modernisation homogénéisante. Cette résurgence de l'histoire est, elle aussi, un aspect écologique, de conservation de la mémoire; c'est en ce sens très riche qu'on a vu s'affirmer au cours de la décennie précédente la centralité du paradigme écologique : cela va aussi naturellement de pair avec la résurgence des moments du corps et de la nature que les deux époques précédentes avaient oubliés en estimant qu'ils avaient été absorbés par le progrès technique. La critique de la modernité est donc bien à l'ordre du jour, mais pas au sens postmoderne : il est bien clair aujourd'hui qu'il faut mettre en discussion le paradigme prométhéique ayant caractérisé la modernité au moins à partir du XVIII ${ }^{\mathrm{e}}$ siècle, un prométhéisme qui voulait anéantir aussi la pluralité des cultures et des traditions tout comme la totalité de la nature. À partir de cela, le paradigme de la différence ou de l'hétérogénéité, déjà central dans la conception postmoderne, assume une nouvelle signification: la culture anthropologique ne pose plus son thème de la diversité à partir du culturalisme, mais en articulant différence et corps, différence et nature : c'est le cas d'anthropologues ou de géographes comme Tim Ingold, Philippe Descola ou Augustin Berque, sur lesquels je reviendrai par la suite en fournissant un peu plus de détails.

Il faut bien être conscients du fait que, derrière ce nouveau paradigme écologique, se produit un puissant changement historique : nous ne sommes plus à l'ère de la domination d'une puissance unique, américaine - même s'il est vrai que les États-Unis ont encore un rôle prééminent dans le monde. Dans l'ensemble, la situation a bien changé : il y a, au niveau géopolitique une pluralité de pôles, et parmi eux, beaucoup sont des pays nouveaux, non occidentaux; la Chine, sans doute, mais, plus en général, ceux que l'on nomme « Brics » (c'est-à-dire Brésil, Russie, Chine, Inde, Afrique du Sud). Après cette globalisation synonyme d'homogénéisation et d'unification unilatérales, on assiste à nouveau à l'explosion de la pluralité et, bien sûr, des conflits. Certes par là, l'histoire actuelle se recoupe avec le discours anticolonial et anti-occidental déjà bien amorcé au cours des années 1960 et 
ensuite repris et développé par le postmodernisme; ce discours implique incontestablement une nouvelle fois la centralité du savoir anthropologique.

$\mathrm{Au}$ vu de tout cela, quel est le rôle de notre Europe ? En apparence, l'Europe semble ne jouer aucun rôle, si l'on considère aussi bien la situation de non-identité politique du continent européen que les choix politiques des groupes qui dominent à Bruxelles. Mais sur les valeurs qui sont à l'ordre du jour - nature, corporéité, vie, différence, marginalité - l'Europe a bien des choses à dire. Le fait que la crise de la pensée unique américaine néolibérale recentre l'attention sur de nombreux thèmes du Kulturpessimismus, de la phénoménologie, de l'École de Francfort, d'ailleurs portés au cœur de ce séminaire, ne fait pas l'ombre d'un doute. Tous ces courants sont typiquement européens, et s'il est vrai que l'idée husserlienne d'Europe est occidentale et eurocentrique, comme cela est évoqué dans d'autres contributions du présent volume ${ }^{1}$, l'enrichissement de ce concept borné d'Europe par l'échange avec les cultures non occidentales est l'enjeu d'un discours phénoménologique entamé depuis des décennies : il suffit de penser à l'essai de Merleau-Ponty De Mauss à Lévi-Strauss et à L'Autre cap de Jacques Derrida $^{2}$, entièrement consacré à la question de l'Europe. Chacun de ces écrits met en valeur le concept d'une Europe ayant sa force et sa richesse dans la pluralité de ses traditions, de ses langues et de ses dialectes, de ses particularités, qui résistent à l'homogénéisation universalisante. Un certain rapport avec la tradition, ou mieux, avec les traditions au pluriel ainsi qu'avec les histoires est définitoire de l'Europe et il se rattache strictement au concept de nature et de corporéité pour constituer ce nouveau paradigme écologique en pleine émergence. Il est aussi évident que l'espace européen et le complexe des cultures européennes, s'ils sont reliés aux traditions non occidentales, à leur valorisation, peuvent donner à ce paradigme naissant un profil bien différent de celui qu'il peut avoir aux États-Unis.

$*$

Avant d'évoquer quelques auteurs dont les travaux actuels développent ce paradigme écologique, je voudrais fournir une ou deux précisions sur le concept de nature repris par Merleau-Ponty à partir de Husserl et développé

\footnotetext{
${ }^{1} C f$. Laurent Perrau, « Husserl critique de la modernité », ce volume.

${ }^{2}$ Cf. Maurice Merleau-Ponty, Signes, Paris, Éditions Gallimard, 1960, p. 143 sq. ; Jacques Derrida, L'Autre cap, Paris, Éditions de Minuit, 1991.
} 
dans ses leçons dont le texte est aujourd'hui à notre disposition ${ }^{1}$. La rencontre entre l'idée merleau-pontienne de nature et l'idée d'écologie, il faut d'abord le dire, n'est pas escomptée : en effet, seul un travail d'interprétation pourra permettre ce croisement. Ne nous surprenons pas, car lorsque Merleau-Ponty tient ses cours, entre 1956 et 1960, sa perspective concerne la crise du système soviétique d'une part, sa rupture avec Sartre et le marxisme d'autre part, une prise de distance rendue manifeste par Les Aventures de la dialectique $^{2}$. Donc, la critique du prométhéisme et du productivisme n'est pas directe, mais sous-jacente à la prise de distance par Merleau-Ponty à la fois par respect du capitalisme et du socialisme ; mais telle critique dérive aussi de son lien avec Lévi-Strauss. Sur le néocapitalisme, sur la grande vague de développement et d'exaltation de la technologie qu'il suscita au début des années 1960 nous trouvons certes des prises de position durant la période 1958-196133, mais sa mort, comme nous le savons, a empêché Merleau-Ponty de développer un projet de nature assurément politique autant que philosophique et ontologique. Toutefois, l'idée merleau-pontienne de nature, telle qu'elle émerge des cours du penseur sur la nature, est pour nous fondamentale; pour reprendre le fil du discours sur la culture des années 1960, avec lequel j'ai débuté mon exposé, on peut mesurer ici les limites de la génération successive des poststructuralistes, lesquels n'ont pas poursuivi cette ligne de réflexion sur la nature si courageusement amorcée par Merleau-Ponty. En quel sens, donc, le concept de nature merleau-pontien estil encore vivant pour nous? Dans le sens, essentiellement, que la nature de Merleau-Ponty n'est pas la wilderness américaine, elle n'est pas cette idée de nature vierge constituant l'autre face de la conception occidentale de culture : tout comme pour sa pensée du corps ou de la chair, son idée de nature veut se situer au-delà de l'opposition cartésienne sujet-objet, âme-corps. En développant la pensée husserlienne sur la réciprocité d'activité et passivité, Merleau-Ponty présente la nature comme un moment d'inclusion de notre action dans un être qui nous précède; au-delà du dialogue avec l'ontologie

${ }^{1} C f$. Maurice Merleau-Ponty, La Nature, Paris, Éditions du Seuil, 1995. Sur l'idée merleau-pontienne de nature, la littérature critique est très vaste: dans la revue Chiasmi International on trouvera une sélection très représentative des études les plus récentes et significatives sur l'argument; en particulier, voir le vol. 2 (2000), intitulé « Merleau-Ponty. De la Nature à l'ontologie » et le vol. 7 (2005), « MerleauPonty. Vie et individuation, avec des inédits de Merleau-Ponty et de Simondon ».

${ }^{2}$ Paris, Éditions Gallimard, 1955.

3 Voir par exemple le cours de 1958-1959 «La Philosophie aujourd'hui», dans Maurice Merleau-Ponty, Notes de cours 1959-1961, Paris, Éditions Gallimard, p. 33 sq. 
heideggérienne, cette idée d'une action qui est en soi essentiellement réception constitue l'élaboration d'une réflexion sur le problème du vivant, central dans la pensée de Merleau-Ponty à son début dans les années 1930 : la relation de l'être vivant avec son milieu, l'idée même de perception contient déjà cet aspect de réciprocité de l'activité et de la passivité comme noyau du concept de nature ${ }^{1}$. Si Merleau-Ponty, dans son cours, revendique la nature comme quelque chose d'irréductible à l'acculturation, à l'historicisation, ce noyau irréductible n'est pas la nature vierge mais le reste, l'altérité absolue par rapport à l'acte d'acculturation qui, selon Merleau-Ponty, doit être rapporté au fait que chaque action du vivant ne se déroule pas dans le vide d'un rien absolu, mais s'insère et présuppose un être, un milieu ; c'est d'ailleurs au même résultat que nous parvenons si nous partons de l'idée du thétique, qui n'est qu'une élaboration d'un moment pré-thétique ne pouvant jamais se réduire à la transparence de la conscience ${ }^{2}$.

Mais on perçoit alors immédiatement que ce concept de nature articulé sur la réciprocité de la passivité et de l'activité a son pendant dans le concept d'institution sur lequel Merleau-Ponty avait donné un autre cours en 1954$1955^{3}$, lorsqu'il avait amorcé une réflexion qui l'aurait conduit à sa dernière vision ontologique. La réciprocité d'activité et de passivité est déjà au centre de cette nouvelle analyse merleau-pontienne sur l'histoire dont le point de départ est la mise en question de l'idée de structure chez Saussure et LéviStrauss ; on ne doit pas séparer l'élaboration de Merleau-Ponty sur la nature de celle concernant l'histoire et l'institution, car le phénomène de la structure implique la même inclusion de l'activité dans la passivité : l'histoire n'est pas un facere, une action unilatérale; il y a reprise, il y a tradition, il y a passivité, il y a reste dans l'histoire. Donc, c'est le même mouvement conceptuel qui nous porte à reconnaître un noyau d'irréductibilité dans la nature et dans l'histoire, à poser des limites au productivisme, et ainsi à soutenir une certaine écologie et, en même temps, à revaloriser les traditions, à nous imposer ce culte des monuments dont parlait, au début du $\mathrm{XX}^{\mathrm{e}}$ siècle, le grand historien de l'art Aloïs Riegl ${ }^{4}$. Ici, on touche à un noyau conceptuel

${ }^{1}$ Cf. Maurice Merleau-Ponty, L'Institution. La passivité. Notes de cours au Collège de France (1954-1955), Paris, Éditions Belin, 2003.

2 À ce propos, voir les remarques d'Annabelle Dufourcq dans son texte sur la distance de Merleau-Ponty par rapport au nihilisme (Annabelle Dufourcq, «La Fin de la crise ? Pour un dépassement phénoménologique du modèle critique », ce volume).

${ }^{3} C f$. Maurice Merleau-Ponty, L'Institution, op. cit.

${ }^{4}$ Cf. Aloïs Riegl, Le Culte moderne des monuments. Son essence et sa genèse, trad. fr. D. Wieczorek, Paris, Éditions du Seuil, 1984. 
qu'il reste encore - en grande partie - à élaborer : sur cette nouvelle relation entre culture et nature, la réflexion est tout à fait actuelle et la problématique appelle sans aucun doute un approfondissement ; mais je crois quand même pouvoir au moins affirmer qu'ici la vieille opposition KulturZivilisation, ainsi que toutes les catégories du pessimisme culturel, avec ses implications critiques envers la technè, l'industrialisation etc., sont remises en question.

Enfin, ce noyau conceptuel nature-culture, activité-passivité, au cœur de la pensée merleau-pontienne sur le phénomène de la vie, peut être considéré de beaucoup d'autres points de vue : nous voilà à nouveau ici confrontés à un programme de recherche très complexe. En premier lieu surgit la question de la vision et de sa relation au phénomène de la vie : vous vous rappellerez de ces passages des cours sur la nature où Merleau-Ponty, sur les traces du grande biologiste Portmann, examine tous ces rituels de la vie des oiseaux dans lesquels la monstration, le fait de s'exposer à la vue des autres, est centrale ${ }^{1}$. Mon intention n'est pas d'affronter le problème de la relation culture animale-culture humaine, animal-homme qui est implicite dans ces passages. Je voudrais davantage insister sur un autre point; en effet, entre la réciprocité de l'activité et de la passivité et la centralité de la vision, il y a un lien essentiel. S'exposer à la vue des autres signifie l'institution de cette relation de réciprocité qui est encore une expression de la relation entre passivité et activité. On voit ici que tous les aspects de la réflexion merleaupontienne communiquent entre eux : 1'analyse de la vie est reliée à celle de l'intersubjectivité, la question activité-passivité nous renvoie au concept maussien de don. Précisément ici nous abordons un second groupe thématique : il s'agit de la relation entre vie et utilité, avec toutes les implications anthropologiques, sociologiques et économiques qui vont de pair. Si l'on rattache le thème maussien du don à une réflexion philosophique sur la vision et la vie, si l'on donne à la réflexion sociologique un fond philosophique, quelles en sont les conséquences? D'un côté, nous voyons Mauss qui rejette l'utilitarisme robinsonien; de l'autre, se trouve l'idée que la vie n'est pas simple autoconservation, mais aussi vision. Autrement dit, la vie a un moment de passivité, de suspension de l'activité, de libre automanifestation, de jouissance de l'autre comme de soi-même ; c'est précisément ce moment qu'on ne peut réduire à une relation instrumentale avec l'objet. Mais, et ce serait ici un moment final dans ce programme de recherche, quelle est la relation de ce moment de la vision avec celui de l'utilité ? S'agit-il d'altérité absolue, comme Derrida l'entendait dans son livre sur le

${ }^{1}$ Cf. Maurice Merleau-Ponty, La Nature, op. cit., p. 244 sq. 
don $^{1}$ ? Au contraire, dans la perspective merleau-pontienne, la vision, la passivité n'est pas l'autre absolu de l'activité, c'est son revers, ou sa racine profonde. Ou encore, activité et passivité forment un chiasme indissoluble ; bien des conséquences politiques en découleraient... Mais il s'agit, je le sais bien, d'un ensemble de questions très vastes, véritablement ouvertes, et que je pose avec la conscience nette d'être loin de savoir y répondre.

Pour terminer, voici quelques aperçus sur les auteurs qui, à partir de perspectives et de disciplines différentes, poursuivent actuellement la direction de ce programme merleau-pontien du nouveau paradigme du corps, de la chair ou de la nature : ces figures sont Tim Ingold ${ }^{2}$, anthropologue écossais dont le point de repère fondamental se trouve dans les conceptions du penseur français; le grand géographe et anthropologue Augustin Berque ${ }^{3}$, dont les recherches portent sur la culture japonaise; et Philippe Descola, professeur d'anthropologie de la nature au Collège de France et qui a livré, dans son ouvrage Par-delà nature et culture ${ }^{4}$, une grande synthèse de la recherche anthropologique à l'heure actuelle. Je signale aussi au passage que dans le numéro 14 de «Chiasmi International», la revue de référence de la recherche merleau-pontienne actuelle, on trouve une interview de Descola sur Merleau-Ponty, ainsi qu'un essai d'Ingold ${ }^{5}$, ce qui confirme que nous avons ici un chantier de recherche bien ouvert.

Économie de temps oblige, je voudrais m'arrêter un peu uniquement sur Tim Ingold, chez qui la question au centre de mon intérêt, concernant la relation nature-culture et un nouveau paradigme de la corporéité, est particulièrement évidente. C'est par la critique aussi bien du positivisme biologiste que du culturalisme qu'Ingold prend ses distances par rapport à l'idée ab-

${ }^{1}$ Cf. Jacques Derrida, Donner le temps. 1. La Fausse monnaie, Paris, Éditions Galilée, 1991.

${ }^{2}$ L'ouvrage fondamental de Tim Ingold est The Perception of the Environment. Essays in Livelihood, Dwelling and Skill, London and New York, Routledge, 2000.

${ }^{3}$ Du point de vue philosophique, l'ouvrage le plus important de ce grand représentant de la géographie phénoménologique est Écoumène. Introduction à l'étude des milieux humains, Paris, Éditions Belin, $2010^{2}$.

${ }^{4}$ Paris, Éditions Gallimard, 2005.

${ }^{5} C f$. Philippe Descola, "L'Ontologie des autres », Entretien réalisé par Davide Scarso sur Maurice Merleau-Ponty, dans Chiasmi International, vol. 14, 2012,, p. 33 sq. et Tim Ingold, "The Atmosphere», dans Chiasmi International, vol. 14, 2012, p. $75 s q$. 
straite d'hérédité ayant dominé la biologie néodarwiniste et l'anthropologie culturaliste : tout comme la relation entre génotype et phénotype n'est pas mécanique - le développement du phénotype dépend d'une complexe interaction entre l'organisme et son milieu — de la même manière une culture ne dépend pas de l'héritage de schémas culturels déjà fixés par la tradition, mais d'un processus d'apprentissage mis en œuvre par l'interaction avec des personnes et des choses: "Les formes et les capacités des êtres humains et d'autres organismes ne doivent pas être attribués, en dernière analyse, à l'hérédité génétique, mais aux potentialités génératives du système évolutif $»{ }^{1}$.

En guise d'exemple concret, dans lequel on reconnaîtra la reprise faite par Ingold des idées maussiennes sur les techniques du corps, on trouve celui de l'enfant qui apprend à marcher: il le fait selon la manière qui est approuvée par sa société, mais ce moment culturel n'est pas superposé de l'extérieur à un moment biologique, qui serait celui d'une capacité à la marche universelle de tous les humains ; ce moment biologique et celui de l'apprentissage culturel fusionnent dans ce moment actif par lequel l'enfant s'approprie et transforme dans la pratique une certaine manière de marcher inculquée par ses parents. La marche n'est donc pas un moment d'acculturation où le corps est réduit à une simple matière : le corps vivant est toujours là, il interagit à chaque moment avec celui qui marche; de ce fait, la socialisation et l'acculturation ne signifient à aucun moment un passage du biologique au culturel, car nous continuons à chaque instant à être biologiques et culturels à la fois, à nous mettre en relation avec le monde et les autres et à transformer activement cette relation au monde qui s'exprime dans la marche et dans toutes les autres relations reliant notre corps au monde et aux autres.

Pour cette conception systémique et relationniste du corps et du milieu, Ingold se réclame de l'ouvrage du grand psychologue écologique James Gibson - qui lui aussi a été influencé par la perspective merleaupontienne - et de Gregory Bateson, dont l'Ecology of the mind et l'idée de danse relationnelle et de résonance de l'organisme avec son milieu, mériterait à son tour d'être rapprochée de la Phénoménologie de la perception. Avec

${ }^{1}$ Tim Ingold, «Evolving skills », dans Hilary Rose \& Steven Rose (ed.), Alas, Poor Darwin. Arguments Against Evolutionary Psychology, London, Jonathan Cape, 2000, p. 234 (la traduction française est la mienne). 
une logique des relations, et des champs de relations, dit encore Ingold, la vieille opposition de la nature et de la culture est complètement dissoute ${ }^{1}$.

Dans un essai très important intitulé Building, dwelling, living ${ }^{2}$, Ingold vient de développer un autre aspect de ses conceptions, particulièrement important du point de vue de la question philosophique de la passivité et de l'activité dont nous parlions il y a peu; et il faut ici remarquer que la référence est, cette fois-ci, Heidegger, en particulier son essai Bâtir habiter penser $^{3}$. Préliminaire et fondateur par rapport à la signification commune de « bâtir », c'est-à-dire l'activité, est le moment de l'« habiter » qui implique notre insertion dans l'Être. Ici, il est presque inutile de le remarquer, Ingold ouvre, en se basant sur Heidegger et sur Merleau-Ponty, une perspective écologique qui est, même du point de vue politique, d'une actualité très vive : à une technologie abstraite, comme celle qui prévaut aujourd'hui, il oppose un skilling, une technologie qui ne réduit pas à néant son milieu, mais qui, au contraire, en développe fidèlement et doucement les tendances implicites de développement. Nous avons donc ici un exemple concret de ce qui peut signifier la conception du renvoi réciproque de l'activité et de la passivité, une conception où convergent Husserl, Merleau-Ponty et Heidegger.

Cette réflexion d'Ingold sur la technique, sur l'opposition entre skill et technology, mériterait elle aussi d'être examinée plus en détail, car il s'agit d'une tentative de sortir de l'opposition entre deux idées : l'une voit dans la technique quelque chose de complètement négatif, l'autre la désigne, en tant que telle, comme un élément positif et porteur de progrès - l'alternative dont s'occupe notre séminaire.

${ }^{1}$ Cf. Tim Ingold, « An Anthropologist Looks at Biology », dans Man, vol. 25, 1990, p. 225.

${ }^{2} \mathrm{Cf}$. Tim Ingold, The Perception of the Environment. Essays in Livelihood, Dwelling and Skill, op. cit., p. 172 sq.

${ }^{3}$ Cf. Martin Heidegger, Essais et conférences, Paris, Éditions Gallimard, 1958, p. $186 s q$. 